\title{
Early predictors of unfavourable outcome in subjects with moderate head injury in the emergency department
}

\author{
A Fabbri, ${ }^{1}$ F Servadei, ${ }^{2}$ G Marchesini, ${ }^{3}$ S C Stein, ${ }^{4}$ A Vandelli'
}

${ }^{1}$ Dipartimento dell'Emergenza, Presidio Ospedaliero MorgagniPierantoni, Azienda Unità Sanitaria Locale di Forlì, Forlì, Italy; ${ }^{2}$ Divisione di

Neurochirurgia per la Traumatologia, Ospedale M. Bufalini, Azienda Unità Sanitaria Locale di Cesena, Cesena, Italy;

${ }^{3}$ Alma Mater Studiorum

Università di Bologna, Bologna, Italy: ${ }^{4}$ Department of

Neurosurgery, University of Pennsylvania School of Medicine, Philadelphia, PA, USA

Correspondence to: Andrea Fabbri, MD,

Dipartimento dell'Emergenza,

Presidio Ospedaliero Morgagni-

Pierantoni, Azienda USL Forlì, Via

Forlanini 34, I - 47100 Forlì, Italy; andrea.fabbri@formulatre.it

Received 10 March 2007 Revised 2 August 2007 Accepted 13 August 2007

Published Online First

31 August 2007

\begin{abstract}
Background: Subjects with moderate head injury are a particular challenge for the emergency physician. They represent a heterogeneous population of subjects with large variability in injury severity, clinical course and outcome. We aimed to determine the early predictors of outcome of subjects with moderate head injury admitted to an Emergency Department (ED) of a general hospital linked via telemedicine to the Regional Neurosurgical Centre.
\end{abstract}

Patients and methods: We reviewed, prospectively, 12675 subjects attending the ED of a General Hospital between 1999 and 2005 for head injury. A total of 309 cases (2.4\%) with an admission Glasgow Coma Scale (GCS) 9-13 were identified as having moderate head injury. The main outcome measure was an unfavourable outcome at 6 months after injury. The predictive value of a model based on main entry variables was evaluated by logistic regression analysis.

Findings: $64.7 \%$ of subjects had a computed tomographic scan that was positive for intracranial injury, $16.5 \%$ needed a neurosurgical intervention, $14.6 \%$ had an unfavourable outcome at 6 months (death, permanent vegetative state, permanent severe disability). Six variables (basal skull fracture, subarachnoid haemorrhage, coagulopathy, subdural haematoma, modified Marshall category and GCS) predicted an unfavourable outcome at 6 months. This combination of variables predicts the 6-month outcome with high sensitivity (95.6\%) and specificity (86.0\%).

Interpretation: A group of selected variables proves highly accurate in the prediction of unfavourable outcome at 6 months, when applied to subjects admitted to an ED of a General Hospital with moderate head injury.

Subjects admitted to an Emergency Department (ED) with moderate head injury (Glasgow Coma Scale (GCS) 13-9) represent a particular challenge for the emergency physician. They are a heterogeneous population with large variability in injury severity, clinical course, neurological recovery and long-term outcome. ${ }^{1-4}$

Injury progression is highly variable; although most subjects recover within several days, many deteriorate and need neurosurgical treatment, and a few will die or develop a permanent vegetative state. The type and severity of intracranial injury dictates neurological and clinical deterioration, but predictors of unfavourable outcome in subjects on admission to ED are not well defined. ${ }^{5}$

In a few cases, injury progression might be extremely rapid and the ultimate outcome will be unfavourable because of delayed diagnosis and surgical treatment ${ }^{6-10}$; in other cases, injury does not progress, and the final outcome is usually favourable.

Emergency physicians are, therefore, asked to take early and rapid decisions regarding possible neurosurgical procedures, type of treatment and level of care. Early predictors of CT progression and subsequent unfavourable outcome have been studied only in selected cohorts of patients admitted to neurosurgical settings and with head injury of variable severity, particularly in subjects with intracerebral injuries ${ }^{11}$ or traumatic subarachnoid haemorrhage (tSAH). ${ }^{12}$ In the study by Chieregato et al, ${ }^{12}$ moderately head-injured patients had a 3-fold mortality increase, whenever there was an associated tSAH on the admission CT.

The aim of this study was to evaluate early predictors associated with unfavourable outcome in subjects with moderate head injury on admission, by reviewing a prospective observational database of subjects attending the ED of a General Hospital without any patients' selection.

\section{PATIENTS AND METHODS \\ Database}

All cases attending the ED of our General Hospital with a moderate head injury are treated according to pre-defined procedures. ${ }^{13} \mathrm{~A}$ number of clinical variables are systematically registered for outcome purposes in each patient according to a specific protocol proposed by the NCWFNS (Neurosurgical Committee of World Federation of Neurosurgical Societies) for subjects with mild head injury and modified for cases with moderate (GCS 13-9) and severe injury (GCS <9). The Head Injury Registry consists of the consecutive medical records of all patients attending the ED of Forlì, Italy, from 1999 for acute head injury within 24 hours from trauma. We define moderate head injury as GCS $13-9^{14}$ and perform early CT in all such cases.

\section{Study population, setting and design}

We present the analysis of our data up to December 2005, when the database included 12715 consecutively triaged cases living in the area of Forlì. In 12242 cases (96.3\%), the head injury was classified as mild (GCS 15-14), in 309 $(2.4 \%)$ as moderate (GCS 13-9), and in 164 (1.3\%) as severe (GCS <9). The ED of Forlì is coupled with a 450 acute bed general hospital, providing general clinical services (except cardiac surgery and neurosurgery). The ED treats about 50000 subjects per year from a population of 171000 inhabitants in an area of $1380 \mathrm{~km}^{2}, 58 \%$ of the population live 
in an urban environment. The hospital treats more than 21000 subjects, with 900 admissions to intensive care. The hospital database is directly connected with the General Register Office of the District.

Eligibility criteria of subjects considered in the present analysis were an acute moderate head injury, defined by admitting GCS of 13-9 in patients seen within 24 hours from any trauma to the head in local inhabitants aged $\geqslant 10$ years. Exclusion criteria were severe hypotension caused by extracranial injuries (systolic blood pressure persistently $<90 \mathrm{~mm}$ $\mathrm{Hg}$ ) (8 cases), need for cardiopulmonary resuscitation (5 cases), and penetrating head injury (2 cases). Also excluded were 14 subjects who needed sedation for intubation before ED arrival. The ED personnel are trained to respond to emergencies according to American Heart Association guidelines. Our final study group included 309 subjects, comprising $91.4 \%$ of all moderate head injury patients.

The final outcome was determined 6 months after injury. First, a systematic search of all patients with intracranial lesions was carried out by checking the death certificates and the medical databases of our local health district. Following this, a member of the ED staff contacted all patients who survived by means of a structured telephone interview, to determine the presence of disability.

On admission to ED, CT scan without contrast was performed with a third-generation equipment, with cuts of $10 \mathrm{~mm}$ or less from the foramen magnum to the vertex, and including both soft tissue and bone windows. CT scans showing intracranial post-traumatic lesions were real-time reviewed by a neurosurgeon of the nearest Neurosurgical Unit (Cesena, $17 \mathrm{~km}$ away), consulted via a teleradiology system. By local protocol, the CT scan had to be performed within 2 hours from ED admission. Following CT scan, patients were either immediately transferred to the Neurosurgical unit (109 cases, $35.3 \%)$, treated in Intensive Care unit $(63,20.4 \%)$ or observed in ED (118, 38.2\%) or General Surgery (17, 5.5\%). Any cases initially observed outside the Neurosurgical unit were transferred to neurosurgery if subsequent findings suggested the need for intervention.

The time interval between trauma and the admission CT scan was recorded for each patient. Also recorded were any subsequent control CT scans, carried out according to clinical and radiological results, within 72 hours from the original event.

Measurements of blood alcohol levels, as well as toxicological tests for drugs and/or drugs of abuse, were performed at the discretion of the physician only in 214 patients (69.3\%).

For the purpose of the present study, the initial and any subsequent CT scans were reviewed retrospectively by two investigators ( $\mathrm{AF}$ and $\mathrm{AV}$ ), and classified according to the criteria of Marshall et $a l^{15}$ modified according to the revision of the European Brain Injury Consortium (EBIC). ${ }^{16}$

The protocol of the present report was carried out according to the Helsinki Declaration and approved by the senior staff Committee of the Azienda Unità Sanitaria Locale of Forlì, a board regulating non-interventional studies and equivalent to an Institutional Review Board.

\section{Variables definition}

In the definition of post-traumatic injuries, we included: (a) depressed skull fracture (DSF); (b) intracerebral haematoma/ brain contusion(s) (ICH); (c) tSAH; (d) subdural haematoma $(\mathrm{SDH}) ;(\mathrm{e})$ epidural haematoma $(\mathrm{EDH})$; and (f) intra-ventricular haemorrhage (IVH) (17). An effort was made to separate diffuse axonal injury patterns from the contusion group.

Injuries were coded by experienced medical personnel according to the Abbreviated Injury Scale (AIS) ${ }^{17}$ for any body region (head-neck, face, chest, abdomen, extremities, external). The Injury Severity Score (ISS) ${ }^{17}$ was calculated as the score derived from the three most severe injuries of each body region. In the analyses, ISS was considered only if $>15$. Admission CT data were available in all patients. The modified Marshall classification was obtained on the basis of the "worst" CT scan as the one recorded under "final CT classification (worst observed)" in the patients' discharge documentation. Data on the worst CT scan were available on all subjects. Haematoma evacuation and/or skull fracture elevation were only considered in the first 7 days after diagnosis. ${ }^{18}$

Field and admission GCS scores were also recorded. In our protocol, GCS score was calculated every 60 minutes after the initial assessment for 2 hours, and every 120 minutes thereafter. In case of clinical deterioration, an additional CT was immediately requested. In our protocol in subjects with ICH, an additional CT scan was performed within 24 hours. Hypotension (systolic blood pressure $<90 \mathrm{~mm} \mathrm{Hg}$, and hypoxia $\left(\mathrm{SpO}_{2} \%<92 \%\right)$ measured at any time were also considered. ${ }^{19}$

Laboratory data were also performed to document blood levels of alcohol and/or drugs of abuse, whenever clinically indicated. Patients' coagulation status (prothrombin time, partial thromboplastin time and platelet count) was carried out by protocol in all cases. Normal values of the International Normalised Ratio (INR) for our laboratory are $<1.4$.

\section{Outcome measures}

The main outcome considered in the analysis was death, a permanent vegetative state or permanent severe disability, measured by the Glasgow outcome scale (GOS) $)^{20}$ score at 6 months, globally defined as unfavourable outcome. For ease of analysis and reporting, the five-point GOS score was categorised as either favourable (moderate disability or good recovery) or unfavourable (dead, vegetative or severe disability). The followup GOS was rated by an expert physician unaware of the study protocol, on the basis of the response to a structured telephone call. ${ }^{18}$

\section{Analyses}

Mean value, standard deviation (SD), median and interquartile range (IOR), and frequencies were used to describe data distribution. Univariate and multivariable stepwise forward logistic regression analysis was used to identify factors related to different outcomes; the goodness of fit of the linear model was tested by the Hosmer-Lemeshow linearity test.

The $t$-test for linear trend in proportion was used to test the linear association between the prevalence of intracranial injury and GCS.

The following variables were tested in multivariable logistic regression analysis: sex, age (as continuous variable), presence of any co-morbidity (history of diabetes, hypertension, coronary artery, respiratory, neurological and gastrointestinal diseases, permanent disability), GCS after ED stabilisation, skull fracture (basal or linear), modified Marshall category, type of intracranial injury (see above), ISS, coagulopathy, alcohol and/or drug intoxication, high-risk dynamics of injury, hypotension and hypoxia.

The odds ratio (OR) and 95\% confidence intervals (95\% CI) were also calculated. A score for risk of unfavourable outcome 
(formula, see Appendix) was calculated for each patient on the basis of the coefficients computed by the logistic regression derived from variables entering the stepwise procedure. The accuracy of such a risk score was then evaluated by the area under the receiver operating characteristic (ROC) curves. ${ }^{21}$ The best cut-off was chosen as the value that maximised the likelihood ratio (LR), computed by the following formula: $L R=$ (probability of true positive + probability of true negative)/ (probability of false positive + probability of false negative).$^{18}$ Sensitivity and specificity were evaluated at best cut-off, as well as the positive predictive value (PPV) and the negative predictive value (NPV). ${ }^{22}$ Statistical analyses were performed running the SPSS/PC+ statistical package on a personal computer. ${ }^{23}$ Twotailed $p$ values less than 0.05 were considered to be statistically significant.

\section{RESULTS}

\section{Database}

The median age of the 309 patients with moderate head injury was 50 years (IOR, 30-75), with 31 (10\%) patients aged 10-20 years and 116 subjects $(37.5 \%) \geqslant 65$ years. Co-morbidities more frequently included coronary artery disease (109 cases, 35.3\%) and neurological diseases (70 cases, $22.7 \%$ ) without differences in relation to outcome. Thirty-four cases $(11.0 \%)$ were on warfarin treatment. The median injury severity score was 14 (IOR 10-22), with significant differences in relation to outcome. In $73 \%$ of subjects with unfavourable outcome, the ISS was $>15$ (severe injury) versus $42 \%$ of subjects with favourable outcome (table 1$)$. The ISS was $>30$ in only 15 cases $(4.8 \%)$.

The median injury-to-door time was $60 \mathrm{~min}$ (IOR, 35-90), and the door-to-CT time was $102 \mathrm{~min}$ (IOR, 74 to 128) without any systematic trend in the period between 1999 and 2005. Adherence to local protocol was nearly complete; an initial CT scan was performed in all cases $(100 \%)$, but the door-to-CT time was $<2$ hours in only 209 cases $(64.7 \%$ ) (table 1$)$.

\section{Intracranial lesions}

Intracranial lesions were detected in 200 subjects (64.7\%). In 191 cases $(61.8 \%)$, the diagnosis was made on the basis of the initial CT scan, and in only 9 cases (2.9\%) was the diagnosis based on a second CT (all these cases had a dubious lesion at first evaluation). A single lesion was present in 81 cases $(40.5 \%$ of positive CT), but in 64 cases $(32.0 \%)$ more than one lesion was diagnosed. An isolated intracranial injury without additional injuries (AIS >1) in other areas was present in 121 subjects (39.1\%). Subjects with intracranial lesions were more likely to be $\geqslant 65$ years ( $42.0 \%$ vs. $29.4 \%$; OR 1.08 ; $95 \%$ CI, 1.06 to $2.87 ; p=0.036)$. A linear skull fracture was diagnosed in 82 cases $(26 \%)$ and a basal skull fracture in 48 subjects (15\%). The prevalence of intracranial lesions increased with decreasing levels of GCS, from $44.2 \%$ in subjects with a GCS of $13-80 \%$ and $90 \%$ in subjects with a GCS of 9 and 10, respectively ( $t$-test for linear trend in proportion, $\mathrm{p}<0.001)$. The most common intracranial lesions in relation to GCS were $\mathrm{ICH}, \mathrm{SDH}$ and tSAH (table 2). Excluding the 29 cases with intracranial injury, for which neurosurgical intervention was mandatory after the initial CT scan, 240/280 cases (77\%) had an additional CT scan within 24 hours (12-24 hours). Repeated CT scan showed a deterioration in Marshall category in $54 / 280$ cases (19.3\%).

\section{Neurosurgical intervention}

Haematoma evacuation and/or depressed skull fracture elevation were performed in $51(25.5 \%)$ of the 200 subjects with positive CT scans. No subjects with negative CT scan at first evaluation had a mass lesion at repeated CT scan needing neurosurgical intervention within 7 days.

A total of 29 cases (9.4\%) also required surgical intervention for orthopaedics, thoracic or abdominal lesions.

\section{Unfavourable outcome}

A complete follow-up was obtained in most cases: 289/309 (93.5\%). Of the remaining 20 cases, 8 patients died due to complications unrelated to head trauma (major orthopaedics injuries, 2 cases; myocardial infarction, 1 stroke, 2; pulmonary embolism, 2; gastrointestinal haemorrhage, 1), 6 were lost to follow-up, and GOS was unreliable in 6 patients, due to either a previous disability (4 cases) or a trauma-related disability independent of head injury (2 cases).

The outcome was unfavourable in 45 cases (14.6\%). Twelve patients died (3.9\%), 5 during the hospitalisation period, 7 during follow-up. Two patients $(0.1 \%$ of total) were left in a permanent vegetative state and $31(10.0 \%)$ were severely disabled. The majority of cases (264 cases, $85.4 \%$ ) had a favourable outcome, with moderate disability being present in 31 cases $(10.0 \%)$. Also the prevalence of unfavourable outcome was extremely variable, and increased with decreasing GCS at entry (from $2.6 \%$ in subjects with a GCS of $13-44.4 \%$ in subjects with a GCS of 9 - $t$-test for linear trend in proportion, $\mathrm{p}<0.001$ ) (table 3).

An unfavourable outcome was rarely observed in subjects with Marshall category I and II, and was much more common in Marshall category IV, V and VI (table 4). In the 51 subjects submitted to neurosurgical intervention for mass lesion, the outcome was related to the progression of lesions. Only 3/28 subjects operated after the initial CT scan had an unfavourable outcome $(10.7 \%)$, compared with $8 / 23$ subjects who underwent

Table 1 Clinical characteristics of subjects with moderate head injury in relation to unfavourable outcome

\begin{tabular}{|c|c|c|c|}
\hline & Unfavourable outcome $(n=45)$ & Favourable outcome $(n=264)$ & p Value* \\
\hline Males & $30(66.7 \%)$ & $194(73.5 \%)$ & 0.368 \\
\hline Age, median (IOR) & $72(30-80)$ & $51(32-72)$ & 0.032 \\
\hline Co-morbidity & $17(38.6 \%)$ & $134(50.6 \%)$ & 0.196 \\
\hline Door-to-CT time (minutes; median (IQR)) & $90(78-122)$ & $103(74-130)$ & 0.380 \\
\hline Injury-to-CT time (minutes; median (IQR)) & $156(121-213)$ & $168(133-216)$ & 0.329 \\
\hline High-risk mechanism ${ }^{\dagger}$ & $32(71.1 \%)$ & $125(47.3 \%)$ & 0.004 \\
\hline Road accidents & $25(56.8 \%)$ & $173(65.3 \%)$ & 0.310 \\
\hline Accident falls & $19(42.2 \%)$ & $90(34.1 \%)$ & 0.314 \\
\hline Injury Severity Score $>15$ & $33(73.3 \%)$ & $111(42.0 \%)$ & $<0.001$ \\
\hline
\end{tabular}

*Mann-Whitney U test or Fisher's exact test: $p<0.05$.

${ }^{\top}$ Classified according to Canadian rules (pedestrian struck by motor vehicle, ejected from a motor vehicle, fall from height $>3$ feet or five stairs). ${ }^{24}$

IQR, interquartile range. 
Table 2 Type of intracranial injury at CT in relation to Glasgow Coma Scale (GCS) in subjects with moderate head injury

\begin{tabular}{llllll}
\hline Type of injury & GCS $\mathbf{9}(\mathbf{n}=\mathbf{4 5})$ & $\mathbf{G C S} \mathbf{1 0}(\mathbf{n}=\mathbf{5 3})$ & $\mathbf{G C S ~ 1 1}(\mathbf{n}=\mathbf{4 6})$ & $\mathbf{G C S} \mathbf{1 2}(\mathbf{n}=\mathbf{5 2})$ & $\mathbf{G C S} \mathbf{1 3}(\mathbf{n}=\mathbf{1 1 3})$ \\
\hline Depressed skull fracture & $5(11.1 \%)$ & $4(7.5 \%)$ & $1(2.2 \%)$ & $1(1.9 \%)$ & $1(0.9 \%)$ \\
Intracerebral haematoma & $28(62.2 \%)$ & $36(67.9 \%)$ & $26(56.5 \%)$ & $26(50.0 \%)$ & $29(25.7 \%)$ \\
Subarachnoid haemorrhage & $21(46.7 \%)$ & $17(32.1 \%)$ & $13(28.3 \%)$ & $7(13.5 \%)$ & $19(16.8 \%)$ \\
Subdural haemorrhage & $24(53.3 \%)$ & $24(45.3 \%)$ & $20(43.5 \%)$ & $14(26.9 \%)$ & $18(15.9 \%)$ \\
Epidural haemorrhage & $2(4.4 \%)$ & $4(7.5 \%)$ & - & $7(13.5 \%)$ & $5(4.4 \%)$ \\
Intraventricular haemorrhage & $9(20.0 \%)$ & $7(13.2 \%)$ & $7(15.2 \%)$ & $4(7.7 \%)$ & $5(4.4 \%)$ \\
\hline
\end{tabular}

a neurosurgical intervention only after a second CT scan and a clinical and radiological evolution (34.8\%; $p<0.048$, Fisher's exact test).

\section{Outcome prediction}

Six out of the 18 variables considered were independently associated with unfavourable outcome and were selected as independent factors at multivariable stepwise logistic regression analysis (table 5), and the Hosmer-Lemeshow test did not reject linearity.

The ROC-type curve of the risk score for unfavourable outcome (death, vegetative state or severe disability), computed on the basis of the coefficients of the logistic regression, is reported in figure 1 . The accuracy in predicting outcome was $0.848 \pm 0.047$ and the best cut-off was identified in a score value ranging between 8.67 and 9.62 , having a sensitivity of $95.6 \%$ and a specificity of $86.0 \%$ (PPV, 99.1\%; NPV, 46.2\%).

\section{DISCUSSION}

Our model shows that emergency physicians may predict with good accuracy an unfavourable outcome in subjects admitted with moderate head injury (only $2.4 \%$ of subjects visited for head injury in an ED, but $22 \%$ of total cases underwent neurosurgical intervention) on the basis of early predictors: GCS after ED stabilisation, Marshall category, SDH, coagulopathy, $\mathrm{SAH}$ and basal skull fracture.

A large number of studies have been published to identify which patients should be managed with different options after moderate head injury. None of these studies refer to databases derived from an ED of a General Hospital without a Neurosurgical Unit, as in our report, but data are not significantly different. The mortality and morbidity of moderate head injury patients have been judged to be of intermediate grade between the minor and severe head injury categories, but published studies largely vary in relation to the setting of diagnosis and treatment, as well as in protocol design. These differences prevent any comparison of different management regimens. Our study shows that subjects admitted to the ED for moderate head injury have a positive head CT scan in $64.7 \%$ of cases, a need for neurosurgery intervention in $16.5 \%$ of cases, and an unfavourable outcome in $14.6 \%$ of cases. A group of 6 selected variables predicts an unfavourable outcome at 6 months. In a prospective study ${ }^{2}$ on 110 cases with moderate head injury admitted to a Trauma Centre with a Neurosurgical Unit, the prevalence of positive CT scan was $61 \%$, no patients with a normal CT scan developed neurological worsening, and the outcome was unfavourable in $8 \%$ of cases. $^{2}$

The most striking result is, however, the large variability of severity, ${ }^{1-4}$ which accounts for a wide variability in outcome. Whereas nearly all subjects with GCS 13 and a negative CT scan fully recover, the outcome of subjects with GCS 9 and positive CT is unfavourable in nearly $50 \%$ of cases. Subjects with moderate head injury, as defined by accepted criteria, represent a grey zone, and GCS it is not likely to accurately predict prognosis. Other scores have been considered-in particular, the Marshall category, which represents a valid complement to GCS, effectively predicting the probability of unfavourable outcome across the whole spectrum (from 1\% in category I to $94 \%$ of cases in category VI). The Marshall category has now been accepted as one of the main predictors of outcome in subjects with head injury and the worst CT scan obtained during the clinical course yields the best discrimination of unfavourable outcome. ${ }^{9}$

The predictive value of GCS might also be biased by the tiny distinction between mild and moderate head injury. In recent years, subjects with GCS 13 have been transferred from the "mild" to the "moderate" category, ${ }^{5}$ but a one-point change may simply depend on the verbal component of the GCS. Finally, time to GCS assessment, sedation and intubation may introduce additional biases in the classification of injured subjects. In our study, GCS 13-9 was only considered in subjects who were stabilised in the ED, and all subjects needing sedatives before intubation were excluded, either on the scene or immediately after ED admission. One patient in four $(25.2 \%)$ had sedation after stabilisation before CT scan and 33 (10.9\%) cases had deterioration in GCS in the range 13-9 during the workup in the ED. In other cases, GCS calculation may also be misleading for alcohol intoxication or abusive drugs intake. In our database, 104 (33.7\%) subjects had high blood alcohol concentration (mean 155 (SD 73) $\mathrm{mg} / \mathrm{dl}$ ) at the evaluation in the ED.

Table 3 Unfavourable outcome in relation to Glasgow Coma Scale (GCS) in subjects admitted to the emergency department after moderate head injury

\begin{tabular}{llll}
\hline \multirow{2}{*}{ GCS } & Total cases $(\%)$ & Unfavourable outcome $(\%)$ & Favourable outcome (\%) \\
\cline { 2 - 3 } $\mathbf{n}=\mathbf{3 0 9}$ & $\mathbf{n}=\mathbf{4 5}$ & $20(44.4 \%)$ & $\mathbf{n}=\mathbf{2 6 4}$ \\
\hline 9 & $45(14.6 \%)$ & $10(18.9 \%)$ & $25(55.6 \%)$ \\
11 & $53(17.2 \%)$ & $10(21.7 \%)$ & $43(81.1 \%)$ \\
12 & $46(14.9 \%)$ & $2(3.8 \%)$ & $36(78.3 \%)$ \\
13 & $52(16.8 \%)$ & $3(2.6 \%)$ & $110(96.2 \%)$ \\
\hline
\end{tabular}


Table 4 Unfavourable outcome in relation to Marshall category in subjects admitted to the Emergency Department after moderate head injury

\begin{tabular}{|c|c|c|c|}
\hline & Total cases & Unfavourable outcome & Favourable outcome \\
\hline Marshall category & $n=309(\%)$ & $n=45(\%)$ & $n=264(\%)$ \\
\hline I & $109(35.3 \%)$ & $1(0.9 \%)$ & $108(99.1 \%)$ \\
\hline ॥ & $96(31.1 \%)$ & $5(5.2 \%)$ & $91(94.8 \%)$ \\
\hline III & $27(8.7 \%)$ & $4(14.8 \%)$ & $23(85.2 \%)$ \\
\hline IV & $10(3.2 \%)$ & $9(90.0 \%)$ & $1(10.0 \%)$ \\
\hline V & $51(16.5 \%)$ & $11(21.5 \%)$ & $40(78.5 \%)$ \\
\hline VI & $16(5.2 \%)$ & $15(93.7 \%)$ & $1(6.3 \%)$ \\
\hline
\end{tabular}

In our study, only $1 \%$ of cases had unfavourable outcome when the CT scan was negative, and the prevalence of full recovery remained relatively high (85\%) in Marshall category III. Thereafter, the prevalence of unfavourable outcome increased significantly in categories IV and VI, but not in category V, provided that subjects were submitted to a neurosurgical intervention. In particular, in Marshall category $\mathrm{V}$, the prevalence of unfavourable outcome dropped to nearly $20 \%$ following neurosurgical intervention, confirming the importance of mass lesion evacuation in these cases. The importance of neurosurgery is also demonstrated by a post-hoc analysis of risk factor, including neurosurgery in the model. Neurosurgery was independently associated with a favourable outcome (OR, $0.03,95 \%$ CI $0.01-0.15 ; p<0.001)$, and the presence of SDH was no longer predictive of outcome.

In previous reports on moderate and severe head injury, the effects of CT changes after a first CT have been extensively investigated. In subjects with moderate and severe head injury, only $4 \%{ }^{25}$ of subjects after normal CT scan at admission evolved to a mass lesion and a new surgical lesion was diagnosed in nearly $15 \%$ of patients. ${ }^{26}$ High-resolution CT scan can identify early small extra cerebral collections at admission that may evolve towards significant haematomas. ${ }^{27}$ We observed CT deterioration only in $3.2 \%$ of cases, but CT deterioration was followed by a marked negative effect on outcome. These findings bring to the attention of Health Care providers the role of immediate surgery in the management of patients with moderate head injury. In the European Union, most of these patients are usually managed outside Neurosurgical Units, ${ }^{28}$ but there are recommendations in favour of either immediate transfer of patients with a positive CT to specialised centres or a telemedicine system with a protocol for repeat of CT scan integrated into head injury guidelines. ${ }^{29}$

Table 5 Risk of unfavourable outcome in patients after moderate head injury predicted by variables included in the local database

\begin{tabular}{llll}
\hline Multivariable analysis & Odds ratio & 95\% Cl & p Value \\
\hline Basal skull fracture & 8.89 & $2.53-31.26$ & $<0.001$ \\
Subarachnoid haemorrhage & 4.50 & $1.73-11.73$ & 0.002 \\
Coagulopathy & 4.48 & $1.35-14.88$ & 0.014 \\
Subdural haematoma & 3.04 & $1.07-8.61$ & 0.037 \\
Marshall category & 1.82 & $1.33-2.50$ & $<0.001$ \\
Glasgow Coma Scale & 0.59 & $0.42-0.83$ & 0.002 \\
\hline
\end{tabular}

Variables not included in the multivariable analysis: depressed skull fracture, intracerebral haematoma/contusion, epidural haematoma, intraventricular haematoma, Injury Severity Score $>15$, age, co-morbidity, alcohol and/or drugs of abuse intoxication, high-risk characteristics of injury, male sex, hypoxia, hypotension, CT scan deterioration during clinical course.

Marshall category and Glasgow Coma Scale were considered to be continuous variables; basal skull fracture, subarachnoid haemorrhage, subdural haematoma and coagulopathy were considered to be dichotomised variables.
Other factors are definitely relevant in dictating outcome, and coagulopathy is a relevant issue for both management and prognosis. In the diagnostic algorithm, coagulopathy (history of bleeding, clotting disorder or current treatment with warfarin) also needs to be considered in subjects with GCS 15 as a main variable necessitating early CT, independently of symptoms. ${ }^{13}$ Controversial outcomes have been reported from clinical studies. A Canadian study documented a 2.7-fold increase in mortality of anticoagulated patients ${ }^{30}$ and other investigators have reported a 3-10 times increased mortality in anticoagulated head-injured patients requiring hospital admission. ${ }^{31-35}$ In a recent study on approximately 20000 subjects with atrial fibrillation, $50 \%$ of whom were treated with warfarin, a 4-fold increase in traumatic ICH was observed compared with patients with a moderate risk for falls. ${ }^{31}$ In a large population study, both age and anticoagulation with warfarin are reported as independent factors of mortality after head injury. ${ }^{36}$ In our setting, coagulopathy accounted for a 4fold risk of unfavourable outcome in these subjects, after GCS and Marshall category, but the exclusion of coagulopathy in the prognostic algorithm did not significantly impair the sensitivity for outcome prediction.

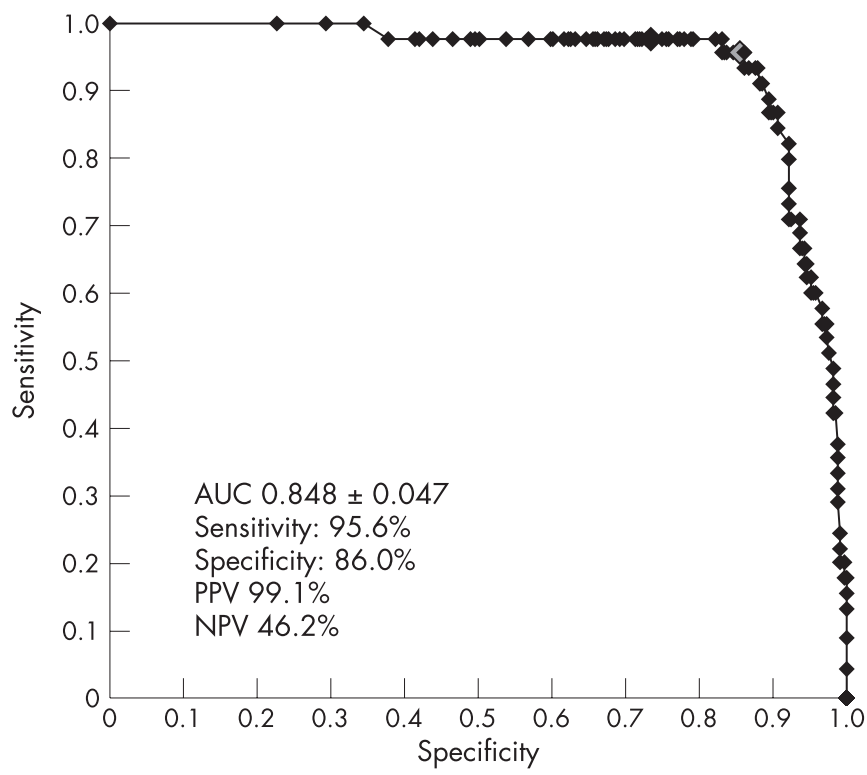

Figure 1 Nonparametric ROC-type plot of the risk score obtained by logistic regression in identifying patients with unfavourable outcome after moderate head injury on the basis of selected variables (basal skull fracture, subarachnoid haemorrhage, subdural haematoma, coagulopathy, Marshall category, Glasgow Coma Scale). The optimal operating point (best cut-off, 8.67-9.62) of the ROC-type curve is also reported. 
The growing elderly population and the expanding indication for anticoagulants are expected to produce more traumatic intracranial injuries, challenging the emergency physicians more and more. In our series, 34 cases (11\%) were treated with anticoagulants, and 32 of them had an INR of more than 2.0 in a population in which $35 \%$ of cases had cardiovascular disease and $37.5 \%$ were $\geqslant 65$ years old. In other settings, these problems may be relatively rare, but there is a need for studies assessing the clinical importance of rapid reversal of anticoagulation.

Surprisingly, our analysis does not identify age as a predictor of outcome, in contrast with a few reports. ${ }^{37}$ This probably depends on the specificity of our aged population and on the selection of variables. Although the severity of lesion may be largely independent of age, the probability of an unfavourable outcome is expected to increase with ageing, but the number of young patients may be too low in our series to give age a statistical significance. In our series, only coagulopathy entered the multivariable analysis among clinical variables, dictating prognosis more than age, but also after the exclusion of coagulopathy, age did not enter the multivariable analysis.

In our database, SAH was the initial diagnosis in 77/309 cases $(24.9 \%)$ and its presence produced a 5 -fold increase in the risk of unfavourable outcome, as previously demonstrated. ${ }^{38}$ The presence of changing lesions in areas of intracerebral lesions on an initial CT scan confirms the role of SAH as an early predictor of evolving brain damage.

Finally, hypoxia and hypotension have also been indicated as early predictors of poor outcome after moderate and severe head injury for the additional risk of secondary brain injury, with hypoxia recorded in $27-55 \%$ of subjects on the scene, in the ambulance or on arrival at the ED. ${ }^{39}$ In our series, hypoxia and hypotension were recorded only in $12 \%$ and $4.9 \%$ of cases, respectively, and did not enter the multivariable analysis.

A few limitations should be acknowledged. The GOS was calculated on the basis of a structured telephone interview, and questionnaires are likely to underestimate rather than overestimate the results, ${ }^{20}$ but telephone-referred disability may be an additional problem. ${ }^{40}$ In addition, the clinical characteristics of subjects with moderate head injury were obtained from variables selected on a prospective database, but all analyses were retrospectively performed: this might bias the results. Finally, the calculation of GCS after ED stabilisation may be a problem. In cases with evolving lesions, GCS at admission might be underestimated and sedative drugs might hamper the GCS calculation, including subjects with severe head injury.

In summary, our data confirm that subjects with moderate head injury have a large variability in prognosis. The prevalence of an unfavourable outcome at 6 months ranges from $2.6 \%$ to $44.4 \%$, and one patient in five needs immediate neurosurgical intervention. In these cases, the detection of early predictors of unfavourable outcome may be important for rapid sequence decisions in the ED. The additional value of SDH, coagulopathy, SAH and basal skull fracture to traditional predictors of GCS and Marshall category are relevant for early prediction of the outcome in these subjects.

\begin{abstract}
Acknowledgements: AF conceived the study, wrote the protocol, coordinated the data collection, interpreted the results and wrote the paper. FS contributed to the interpretation of the results and critical review of the paper. GM contributed to the study design, interpretation of the results and co-wrote the paper. SCS contributed to the interpretation of the results, critical review of the paper and co-wrote the paper. AV contributed to the study design, study coordination, interpretation of the results and critical review of the paper. All authors approved the final version of the paper We are grateful to the Emergency Medical System personnel for helpful support.
\end{abstract}

Competing interests: None.

\section{REFERENCES}

1. Rimel R, Giordani B, Barth B, et al. Moderate head injury: completing the clinical spectrum of brain trauma. Neurosurgery 1982;11:344-51.

2. Fearnside $\mathbf{M}, \mathbf{M c D o u g a l l} P$. Moderate head injury: a system of neurotrauma care. Aust. N Z Surg 1998;68:58-64.

3. Stein SC, Ross SE. Moderate head injury: a guide to initial management. J Neurosurg 1992; 77:562-4.

4. Vitaz TW, Jenks J, Raque GH, et al. Outcome following moderate traumatic brain injury. Surg Neurol 2003;60:285-91.

5. Stein SC. Minor head injury: 13 is an unlucky number. J Trauma 2001:50:759-60.

6. Oertel M, Kelly DF, McArthur D, et al. Progressive hemorrhage after head trauma: Predictors and consequences of the evolving injury. J Neurosurg 2002;96:109-16.

7. Patel NY, Hoyt DB, Nakaji P, et al. Traumatic brain injury: Patterns of failure of nonoperative management. J Trauma 2000;48:367-74.

8. Servadei F, Nanni A, Nasi MT, et al. Evolving brain lesions in the first 12 hours after head injury: Analysis of 37 comatose patients. Neurosurgery 1995;37:899-906.

9. Servadei F, Nasi MT, Giuliani G, et al. CT prognostic factors in acute subdural haematomas: The value of the worst CT scan. Br J Neurosurg 2000;14:110-6.

10. Servadei F, Antonelli V, Giuliani G, et al. Evolving lesions in traumatic subarachnoid hemorrhage: Prospective study of 110 patients with emphasis on the role of ICP monitoring. Acta Neurochir 2002;81(Suppl):81-2.

11. Chang EF, Meeker M, Holland MC. Acute traumatic intraparenchymal hemorrhage: risk factors for progression in the early post-injury period. Neurosurgery 2006;58:647-56.

12. Chieregato A, Fainardi E, Morselli-Labate AM, et al. Factors associated with neurological outcome and lesion progression in traumatic subarachnoid hemorrhage patients. Neurosurgery 2005;56:671-80.

13. Servadei F, Teasdale G, Merry G, on behalf of the Neurotraumatology Committee of the World Federation of Neurosurgical Societies. Defining acute mild head injury in adults: a proposal based on prognostic factors, diagnosis and management. J Neurotrauma 2001;18:657-64.

14. Stein SC, Ross SE. Mild head injury: a plea for routine early CT scanning. J Trauma 1992;33:11-3.

15. Marshall LF, Marshall SB, Klauber MR, et al. A new classification of head injury based on computerized tomography. J Neurosurg 1991;75(Suppl):S14-20.

16. Servadei F, Murray GD, Penny K, et al. The value of the "worst" computed tomographic studies of moderate and severe head injury. Neurosurgery 2000;46:70-7.

17. Baker S, O'Neill B, Haddon W Jr. The Injury Severity Scale: a method for describing patients with multiple injuries and evaluating emergency care. J Trauma 1974;14:187-96.

18. Fabbri A, Servadei F, Marchesini G, et al. Prospective validation of a proposal for diagnosis and management of patients attending the emergency department for mild head injury. J Neurol Neurosurg Psychiatry 2004;75:410-6.

19. Chi JH, Knudson MJ, Vassar MJ, et al. Prehospital hypoxia affects outcome in patients with traumatic brain injury: a prospective multicenter study. J Trauma 2006;61:1134-41.

20. Thornhill S, Teasdale GM, Murray GD, et al. Disability in young people and adults one year after head injury: prospective cohort study. BMJ 2000;320:1631-5.

21. Zweig MH, Campbell G. Receiver-operating characteristic (ROC) plots: a fundamental evaluation tool in clinical medicine. Clin Chem 1993;39:561-77.

22. Hanley JA, McNeil BJ. The meaning and use of the area under receiver operating characteristic (ROC) curve. Radiology 1982;143:29-36.

23. Norusis MJ, SPSS Inc. SPSS/PC+. Base System and Advanced Statistics User's Guide. Chicago, Illinois: Version 5.0. SPSS Inc., 1992.

24. Stiell IG, Wells GA, Vandemheen K, et al., for the CCC Study Group. The Canadian CT head rule for patients with minor head injury. Lancet 2001;357:1391-6.

25. Lobato RD, Sarabia R, Rivas JJ, et al. Normal computerized tomography scans in severe head injury: Prognostic and clinical management implications. J Neurosurg 1986;65:784-9.

26. Roberson FC, Kishore PR, Miller JD, et al. The value of serial computerized tomography in the management of severe head injury. Surg Neurol 1979;12:161-7.

27. Smith HK, Miller JD. The danger of an ultra-early computed tomographic scan in a patient with an evolving acute epidural hematoma. Neurosurgery 1991;29:258-60.

28. Patel HC, Bouamra 0, Woodford $\mathrm{M}$ et al., on behalf of the Trauma Audit and Research Network. Trends in head injury outcome from 1989 to 2003 and the effect of neurosurgical care: an observational study. Lancet 2005;366:1538-44.

29. Servadei F, Antonelli V, Mastrilli A, et al. Integration of image transmission into a protocol for head injury management: a preliminary report. Br J Neurosurg 2002;16:36-42.

30. Lavoie A, Ratte S, Clas D, et al. Preinjury warfarin use among elderly patients with closed head injuries in a trauma center. J Trauma 2004;56:802-7.

31. Gage BF, Birman-Deych $\mathrm{E}$, Kerzner $\mathrm{R}$, et al. Incidence of intracranial haemorrhage in patients with atrial fibrillation who are prone to fall. Am J Med 2005;118:612-7.

32. Ferrera PC, Bartfield JM. Outcomes of anticoagulated trauma patients. Am J Emerg Med 1999;17:154-6.

33. Karni A, Holtzman R, Bass $T$, et al. Traumatic head injury in the anticoagulated elderly patient: a lethal combination. Am Surg 2001;67:1098-100.

34. Mina AA, Knipfer JF, Park DY, et al. Intracranial complications of preinjury anticoagulation in trauma patients with head injury. J Trauma 2002;53:668-72.

35. Gittleman AM, Ortiz AO, Keating DP, et al. Indications for CT in patients receiving anticoagulation after head trauma. Am J Neuroradiol 2005;26:603-6.

36. Franko J, Kish KJ, O'Connell BG, et al. Advanced age and preinjy warfarin anticoagulation increase the risk of mortality after head injury. J Trauma 2006;61:107-10.

37. Stein SC. Outcome from moderate head injury. In: Narayan R, Wilberg J, Povlishock J, eds. Neurotrauma. New York, McGraw-Hill, 1996:755-65. 
38. Servadei F, Murray GD, Teasdale GM, et al. Traumatic subarachnoid hemorrhage: demographic and clinical study of 750 patients from the European brain injury consortium survey of head injury. Neurosurgery 2002;50:261-7.

39. Fearnside MR, Cook RJ, McDougall $P$, et al. The Weastmead Head Injury Project outcome in severe head injury: a comparative analysis of pre-hospital, clinical and CT variables. Br J Neurosurg 1993;7:267-79.

40. Teasdale GT, Pettigrew LEL, Wilson JTL, et al. Analysing outcome of treatment of severe head injury: a review and update on advancing use of the Glasgow outcome scale. J Neurotrauma 1998;15:587-97.

\section{APPENDIX}

Formula for calculating the scores derived from the stepwise logistic regression: Score for unfavorable outcome $=$

$($ FRAT*2.1854)+(tSAH*1.5048)+(COA*1.4991)+(SDH*1.1106)+(MARS*0.6012)+(GC $\mathrm{S}^{*}-0.5272$

FRAT, basal skull fracture; tSAH: traumatic subarachnoid haemorrhage; SDH: subdural haematoma; MARS, Marshall-modified category; GCS, Glasgow coma score. FRAT, tSAH, SDH: 0 if absent or 1 if present.

\section{Neurological picture}

\section{Varicella zoster virus and intracranial dolichoectasia in a late adult cancer survivor}

A 50-year-old secretary presented with a 23 year history of forgetfulness and leftsided deafness following chemotherapy for Hodgkin's lymphoma, complicated by disseminated tuberculosis and varicella zoster virus (VZV). MRI revealed carotid and vertebro-basilar dolichoectasia, and multiple small deep infarcts including a left ventral cochlear nucleus infarct resulting in memory impairment and left sensory neural deafness (figs 1,2).

Intracranial arterial dolichoectasia (IADE) is due to elongation and fusiform dilatation of the intracranial arteries. It is associated with older age, male sex, hypertension and previous history of myocardial infarction, but not with markers of carotid atherosclerosis, and is usually the result of a non-atherosclerotic disorder of the elastic tissue in the arterial wall. ${ }^{1}$ Complications include damage to perforating arteries, lacunar infarction, haemorrhage, brainstem and cranial nerve

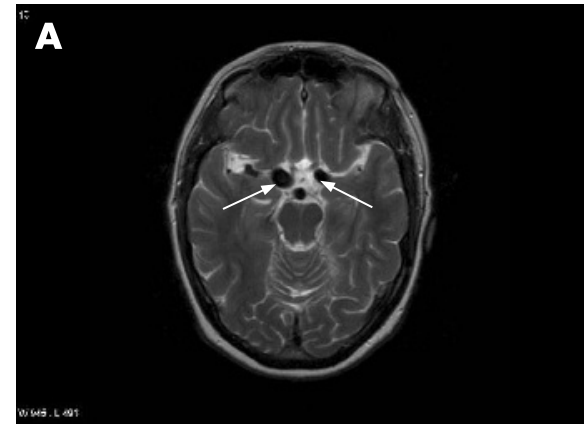

Figure 1 (A) T2 weighted axial MRI showing bilateral terminal carotid artery dolichoectaseia. (B) Left pontine ventral cochlear nucleus infarct.

compression, and obstructive hydrocephalus. $^{12}$

IADE also occurs in association with elastic tissue disorders and Fabry's disease, and also in immunocompromised children with AIDS as a result of VZV angiitis. ${ }^{3} 4$

An occlusive focal VZV angiitis causing stroke after zoster infection in otherwise normal adults is well recognised, ${ }^{4}$ and an acute disseminated VZV cerebral vasculopathy with multiple aneurysms has been reported in an HIV immunocompromised adult. ${ }^{5}$ However, to our knowledge, IADE has not previously been reported in late adult survivors of cancer, immune suppression and VZV infection.

\section{M Dalton, ${ }^{1}$ H R Jäger, ${ }^{2}$ N A Losseff, ${ }^{1}$ R J Greenwood ${ }^{1}$}

${ }^{1}$ Acute Stroke and Brain Injury Service, National Hospital for Neurology and Neurosurgery, London, UK; ${ }^{2}$ The Lysholm Department of Neuroradiology, National Hospital for Neurology and Neurosurgery, London, UK

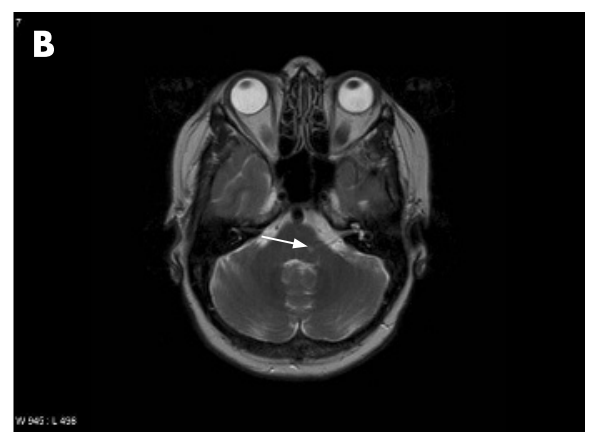

Figure 2 Three dimensional time-of-flight angiography showing ectasia of the left vertebral artery, basilar artery, proximal and intracerebral internal carotid arteries, both M1 segments and right $A 1$ segment, and left $A 1$ segment hypoplasia.

Correspondence to: Dr C M Dalton, Acute Stroke and Brain Injury Service, The National Hospital for Neurology and Neurosurgery, London WC1N 3BG, UK; catherine.dalton@uclh.nhs.uk

Competing interests: None.

J Neurol Neurosurg Psychiatry 2008;79:573. doi:10.1136/jnnp.2007.120725

\section{REFERENCES}

1. Pico F, Labreuche J, Touboul PJ, et al, for the GENIC Investigators. Intracranial arterial dolichoectasia and its relation with atherosclerosis and stroke subtype. Neurology 2003;61:1736-42.

2. Passero SG, Calchetti B, Bartalini S. Intracrania bleeding in patients with vertebrobasilar dolichoectasia. Stroke 2005;36:1421-5.

3. Dubrovsky T, Curless R, Scott G, et al. Cerebral aneurysmal arteriopathy in childhood AIDS. Neurology 1998:51:560-5.

4. Gilden DH, Kleinschmidt-DeMasters BK, LaGuardia JJ, et al. Neurologic complications of the reactivation of varicella-zoster virus. N Engl J Med 2000;342:635-45.

5. Saraya T, Shimura C, Wada H, et al. Evidence for vascular spread of varicella zoster-associated vasculopathy. Ann Intern Med 2006;144:535-7. 